\title{
Aporte de la integración de sistemas de gestión al mejoramiento de la calidad educativa de los colegios maristas de Colombia*
}

\author{
Contribution of Management Systems Integration to the \\ Improvement of Marist Schools Educational Quality in Colombia
}

Contribuição de Inteǵração de Sistemas de Gestão à melhoria
da qualidade educacional dos Colégios Maristas de Colômbia

Recibido: 11 de marzo de 2019 Revisado: 13 de mayo de 2019 Aceptado: 3 de junio de 2019

José Rodrigo Martíne: Narváez**

Universidad Santo Tomás, Colombia

Ingrid Carolina Moreno***

Universidad Santo Tomás, Colombia

Cómo citar este artículo: Martínez Narváez, J. R. \& Moreno, I. C. (2020). Aporte de la integración de sistemas de gestión al mejoramiento de la calidad educativa de los colegios maristas de Colombia. Signos. Investigación en Sistemas de Gestion, 12(1), 137-150. DOI: https://doi.org/10.15332/24631140.5425

Artículo de resultado de investigación.

** Licenciado en Teología. Especialista en Administración y Gerencia de Sistemas de Gestión de Calidad. Universidad Santo Tomás. Correo electrónico: Rodrigomartinezfms@hotmail.com. Orcid: https://orcid.org/0000-0002-9352-6399

*** Administradora de Empresas. Especialista en Administración y Gerencia de Sistemas de Gestión de Calidad. Magíster en Gestión Documental y Administración de Archivos. Docente investigadora del convenio USTA-Icontec, en la línea de investigación de Gestión Integral. Auditora y consultora en sistemas de gestión. Correo electrónico: ingridcmoreno@usantotomas.edu.co, cmrcalidad21@gmail.com. Orcid: https://orcid. org/0000-0003-3624-0877 


\section{RESUMEN}

El mejoramiento en la calidad educativa ha tomado especial relevancia en los últimos tiempos, lo que ha orientado a las instituciones educativas a incorporar en su sistema educativo la posibilidad de gestionar sus actividades a partir de sistemas de gestión como herramientas que favorezcan el logro de los objetivos trazados. La investigación centra su interés en diseñar una propuesta metodológica para la integración de sistemas de gestión basados en los requisitos de las normas técnicas NTC ISO 9001:2015 y la NTC ISO 1400 en los colegios maristas de Colombia, que contribuya a continuar mejorando sus prácticas educativas y a su vez adoptar un sistema integrado de gestión que, a mediano plazo, pueda implementarse y ser certificado. Para la recolección de la información se llevaron a cabo encuestas diagnósticas construidas a partir de la estructura de los requisitos establecidos en las normas técnicas objeto de estudio, aplicada a seis (6) de los siete (7) colegios privados de la comunidad, en los que se tiene la experiencia de la implementación del SGC. A partir del análisis que se realiza, se desarrolla una propuesta metodológica para la integración y posteriormente se valida la propuesta a través del grupo focal con expertos.

Palabras clave: gestión de calidad, gestión ambiental, sistemas integrados de gestión, calidad en la educación.

\section{ABSTRACT}

The improvement in educational quality has taken special relevance in recent times, which has led educational institutions to incorporate into their education system the possibility of managing their activities from management systems as tools that favor the achievement of the objectives stablished. The research focuses on designing a methodological proposal for management systems integration based on the requirements of the technical standards Colombian Technical Standard (Norma Técnica Colombiana, NTC) ISO 9001:2015 and NTC ISO 1400 in the Marist schools of Colombia, which helps to continue improving their educational practices and in turn to adopt an integrated management system that, in the medium term, can be implemented and certified. For the information collection, diagnostic surveys were carried out based on the structure of the requirements established in the technical standards under study, applied to six (6) of the seven (7) private schools of the community, in which you have the experience of implementing the Quality Management System (QMS). Based on the analysis carried out, a methodological proposal for integration is developed and subsequently the proposal is validated through a focus group with experts.

Keywords: quality management, environmental management, integrated management systems, quality in education.

\section{RESUMO}

A melhoria da qualidade educacional ganhou especial relevância com o passar do tempo, levando às instituições educacionais a incorporarem no seu sistema educacional a possibilidade de gerir suas atividades a partir de sistemas de gestão como ferramentas que que favoreçam a conquista dos objetivos delineados. A pesquisa coloca 0 seu interesse na projeção de uma proposta metodológica para a integração de sistemas de gestão baseados nos requisitos das normas técnicas NTC ISO 9001: 2015 e a NTC ISO 1400 nos Colégios Maristas da Colômbia, que contribua a seguir melhorando suas práticas educacionais e, por sua vez, implementar um sistema integrado de gestão que, a médio prazo, possa ser implementado e 
certificado. Para a coleta das informações foram realizadas pesquisas diagnósticas feitas a partir da estrutura dos requisitos estabelecidos nas normas técnicas que são objeto de estudo, aplicada a seis (6) dos sete (7) colégios privados da comunidade, nos quais existe a experiência da implementação do SGC. A partir da análise realizada, desenvolve-se uma proposta metodológica para a integração e em seguida valida-se a proposta através de grupo focal com especialistas.

Palavras-chave: gestão de qualidade, gestão ambiental, sistemas integrados de gestão, qualidade na educação.

\section{INTRODUCCIÓN}

La sociedad ha ido evolucionando en muchos sentidos $\mathrm{y}$, a la par de este proceso, las organizaciones han integrado a sus actividades nuevas dinámicas, buscando estrategias y metodologías que les permitan alcanzar más eficazmente sus objetivos, agregando valor a sus productos o servicios prestados, con el ánimo de continuar vigentes en mercados emergentes y cambiantes. En el ámbito educativo la situación no es muy diferente, como lo manifiestan Fabela \& García (2014), las escuelas y universidades de todo el mundo, están adoptando características de una empresa en la que se aplican distintas reformas con el propósito de mejorar y asegurar la calidad educativa, una de ellas es la implementación de sistemas de gestión, que faciliten el logro de los objetivos planificados.

En el contexto colombiano, el panorama en torno a gestionar la calidad en la prestación de los servicios educativos no es muy distante de lo que sucede en el contexto global. Las organizaciones siguen buscando herramientas que les permitan gestionar de forma más eficaz y efectiva sus actividades, generando impactos positivos en la percepción de sus clientes. Desde la academia se han realizado investigaciones que buscan favorecer estos procesos, como es el caso de Valois Cubillos (2014), quien en su investigación propone una metodología práctica para la integración de la gestión, al considerar a las organizaciones como sistemas conformadas por varios subsistemas, con un interés más allá del cumplimiento de requisitos, presentándose como una alternativa para mitigar la incertidumbre producida por la diversidad de restricciones presentes en la concepción y dirección de las empresas en estos momentos de globalización, competitividad, innovación y lucha por la sostenibilidad.

En nuestro país las instituciones educativas se encuentran expuestas a nuevas exigencias globales, que les obligan revisar y ajustar sus procesos con el fin de ofrecer calidad educativa que contribuya a la formación integral y que posibilite a los estudiantes enfrentarse a la realidad de la sociedad, es decir, que les otorgue las herramientas necesarias para vivir y convivir en sociedad. Desde el Ministerio de Educación Superior (MEN, 2010), se entiende la calidad educativa como aquella que busca que todos los estudiantes, sin importar su procedencia o situación socioeconómica, tengan la oportunidad de adquirir conocimientos y competencias, alcanzar el desarrollo personal y que a partir de ella puedan formarse como mejores seres humanos, ciudadanos con valores éticos, respetuosos de lo público, que ejercen los derechos humanos, cumplen sus derechos humanos y conviven en paz. Esto implica una nueva mirada a la educación, como lo recuerda la Oficina Regional de Educación para América Latina y el Caribe (Unesco, 2017), y supone indudablemente un gran reto para las instituciones de educación, que deben a su vez garantizar procesos formativos de calidad y facilitar el desarrollo de competencias y habilidades personales y profesionales. 
En esta misma línea de análisis sobre la calidad en la educación, Quiroga \& Rodríguez (2014) plantean para alcanzar este objetivo, hay que buscar mecanismos que permitan que el sistema educativo pueda garantizar a los ciudadanos la posibilidad de acceder a una formación de calidad, que permite el desarrollo de competencias para desempeñarse eficazmente en la sociedad del conocimiento, participando en la solución de las problemáticas en el orden de lo regional, nacional e internacional.

Se puede decir entonces que el mejoramiento o aseguramiento de la calidad es uno de los principales retos para el crecimiento y desarrollo de nuestro país y busca que el estado y las instituciones garanticen que los colombianos adquieran conocimiento, destrezas y habilidades que les permitan tener una inserción positiva en el mundo laboral cada vez más competitivo y que es deber del Estado el garantizar que los contenidos y la operativización de la calidad en los planteles educativos sean realizadas responsablemente y además sean pertinentes con las necesidades y exigencias de los distintos sectores. En este sentido, se ve cómo desde el Ministerio de Educación Nacional MEN (2016) se han generado directrices buscando el desarrollo de competencias en los estudiantes y el aseguramiento de índice sintético de calidad, mediante la implementación de normas de gestión y la generación de políticas que regulan la prestación del servicio educativo.

Desde el Estado se han constituido políticas para que las personas puedan acceder a una educación de mayor calidad y donde el Estado aparece como garante del cumplimiento de este derecho. En este sentido, la Ley 115 de 1994, Ley General de la Educación, en el artículo 74 menciona que el Ministerio de Educación Nacional establecerá y reglamentará un Sistema Nacional de Acreditación de la Calidad de la educación formal y no formal, con el fin de garantizar a la familia, a la sociedad y al Estado que las instituciones educativas cumplen con los requisitos de calidad y desarrollan los fines propios de la educación.

Los colegios maristas forman parte de una organización dedicada a una labor tan importante como es la educativa, que en los últimos tiempos se ha dado a la tarea de estandarizar sus procesos a través de la estructuración e implementación de un sistema de gestión de la calidad que permita desarrollar procesos institucionales de calidad, con un enfoque administrativo de mejoramiento continuo, cubriendo el proceso administrativo de planear, organizar, dirigir y controlar a partir del sistema en mención, alcanzado la efectividad organizacional en la prestación de un servicio de calidad.

En efecto, el propósito principal de la investigación es generar una propuesta metodológica que facilite en la organización la integración y que a su vez provea herramientas prácticas que contribuyan a la cultura de la calidad, al crecimiento organizacional, a la estandarización de procesos y a la sostenibilidad de la organización.

Actualmente a nivel global se viene presentado la tendencia en las organizaciones tanto del sector privado como del sector público de integrar a sus actividades distintos modelos de gestión. Como lo expresan Heras Saizarbitoria, Merce \& Casadesus (2007), la gran incidencia que en los últimos años ha tenido la aparición y difusión de diferentes estándares de sistemas de gestión en las organizaciones ha servido como referente en la estructuración de la plataforma estratégica, en el desarrollo de los procesos y en el establecimiento de dinámicas que propendan por la mejora continua.

Por consiguiente, los modelos de gestión se han convertido para las organizaciones en referentes estratégicos, que como lo expresa González (2014) permiten afrontar 
con éxito los retos del presente siglo, facilitando establecer acciones de mejora continua que apalanquen el logro, no solo los objetivos estratégicos, sino además la excelencia en los productos o servicios ofrecidos.

Ahora bien, en el análisis de las actividades y el servicio educativo que se presta en los colegios maristas de Colombia se presenta actualmente la necesidad de realizar el proceso de transición de la norma NTC ISO 9001:2008 a la NTC ISO 9001:2015 y a la vez hacer la integración con la norma NTC ISO 14001:2015 para potenciar buenas prácticas que contribuyan a la protección y cuidado del medio ambiente, asumiendo el reto de tener una adecuada relación con el medio ambiente. Se identifica, en la organización que, aunque hay pequeñas acciones para gestionar el medio ambiente en cada uno de los colegios, existe ausencia de una gestión ambiental sistemática y organizada que garantice responder mejor a las exigencias y necesidades de la población con la que se trabaja y a su vez facilite asegurar sus procesos para evitar contingencias en el orden de lo ambiental que se conviertan en obstáculo para alcanzar los fines organizacionales.

La finalidad de la investigación es proponer una metodología orientada a la integración de los sistemas de gestión fundamentados en las normas NTC ISO 9001:2015 y NTG ISO 14001:2015 en el Sistema de Gestión de la Calidad de los Colegios Maristas de Colombia, que contribuya a alcanzar los estándares de calidad esperados, se fortalezca la formación ambiental de los estudiantes en miras al desarrollo sostenible y al buen uso de los recursos institucionales, favoreciendo los procesos de mejorar y el posicionamiento de las instituciones en relación con el servicio educativo que ofrecen a la comunidad.

\section{METODOLOGÍA}

La investigación se realiza en una organización que cuenta con certificación en Sistemas de gestión de calidad y donde en este momento se presentan la necesidad de hacer la transición a la NTC ISO 9001:2015 y la adopción de un sistema de gestión integrado que tenga en cuenta la NTC ISO 14001:2015 para mejorar la calidad del servicio que se presta, para ello se hace uso de herramientas que provee la investigación de carácter cualitativo basada en la lógica y el proceso inductivo, considerada como un medio que permite profundizar en la realidad de la organización y que junto a las herramientas propias del estudio exploratorio posibilita examinar y evidenciar lo que se necesita en la organización para efectuar la transición e integración de sistemas de gestión de la calidad, generando así una perspectiva teórica a partir de este proceso. Sampieri (2010) manifiesta que la metodología cualitativa busca describir, comprender e interpretar los fenómenos, a través de las percepciones y significados de las personas, que en este caso han participado en la construcción e implementación de un sistema de gestión de la calidad en la organización.

En efecto, como también lo manifiestan Bonilla \& Rodríguez (1995), esta metodología permite profundizar en el problema de estudio, con base en el análisis de la percepción de la realidad y la forma como interpretan las diferentes variables que impactan, en este caso un sistema de gestión de la calidad, como son las personas que participan de su planificación, implementación, mantenimiento y mejora continua. Otro aspecto que determina el carácter cualitativo de esta investigación, como lo indican Barrón \& D'aquino (2007), es que se parte de datos reunidos en forma directa por el investigador para realizar el respectivo análisis, deducción y posterior interpretación de la información. En este sentido, el enfoque cualitativo permite indagar y hacer 
procesos descriptivos que van de lo particular a lo general, facilitando el análisis, la comprensión y generar conocimiento a través del desarrollo del proceso investigativo (Sampieri, Fernández \& Baptista 2010).

\section{Fases de la investigación}

El proceso investigativo se realiza en cuatro momentos, adaptando en este caso las fases que Hernández (2014) propone para dar cumplimiento a cada uno de los objetivos propuestos en la investigación.

Fase 1: Planeación o proyectiva. Desarrollo de la planificación, se determina el campo de estudio de la investigación, planteamiento del problema, antecedentes, objetivos y base teórica y conceptual en la que se sustentará el proceso investigativo.

Fase 2: Metodológica. Es el momento de la elección de un método y las estrategias que guiarán el proceso investigativo. Corresponde al primer objetivo, en él se determina la población en la que se va a realizar el análisis, se eligen los instrumentos y técnicas necesarias para recolectar los datos, que en este caso serán las encuestas diagnósticas de la NTC ISO 9001:2015 y la NTC ISO 14001:2015, proporcionadas por el Convenio USTAIcontec, encuestas aplicadas a la líder del sistema de gestión de calidad y a los gestores de calidad en seis (6) de los siete (7) colegios privados de la comunidad de Hermanos Maristas, objeto de estudio: Colegio San José de Armenia, Colegio San Luis Gonzaga de Cali, Colegio Champagnat de Ibagué, Colegio Champagnat de Ipiales, Instituto Champagnat de Pasto y Colegio Champagnat de Popayán.

Fase 3: Técnica. Se procede a la elaboración de la propuesta metodológica de integración con los programas, procedimientos y demás orientaciones necesarias para realizar la integración de los sistemas en la organización. En esta fase se identifican los componentes de cada norma, y desde la estructura del PHVA (planear, hacer, verificar y actuar) se van identificando y elaborando distintas estratégicas para hacer posible dicha integración.

Fase 4: Teórico o sintético. Para efectos de la investigación, en esta fase se buscar dar cumplimiento al tercer objetivo, y para ello se realiza una aplicación simulada de la propuesta y presentación a un grupo de expertos del colegio Champagnat de Ibagué y la líder de calidad de los colegios maristas de Colombia, utilizando la aplicación simulada a través de la metodología del Abaco de Regnier.

\section{RESULTADOS}

En el desarrollo del primer momento de la investigación se aplicó una encuesta diagnóstica en la institución, basada en las normas técnicas NTC ISO 9001:2015 y NTC ISO 14001:2015. Los resultados de las encuestas aplicadas en la organización permitieron realizar un diagnóstico general de la gestión que se viene adelantando en los colegios. De esta manera, se puede establecer cuáles son los aspectos de estos sistemas de gestión que deben ajustarse y fortalecerse, de acuerdo con los requerimientos de las normas para realizar el proceso de transición de la NTC ISO 9001:2008 a la NTC ISO 9001:2015, como también qué aspectos deben tenerse en cuenta en la integración de la SGC y SGA en los procesos y prácticas actualmente ejecutados. En la tabla 1 se observan los resultados de cómo se encuentran cada una de las instituciones respecto a los requerimientos de las normas objeto de estudio. 
Tabla 1. Diagnóstico comparativo del estado de cumplimiento de requisitos del sistema de gestión de calidad basado en la NTC ISO 9001: 2015 en los colegios maristas de Colombia

\begin{tabular}{|l|c|c|c|c|c|c|c|c|}
\hline $\begin{array}{c}\text { Requisitos } \\
\text { de la norma }\end{array}$ & $\begin{array}{c}\text { Colegio } \\
\text { Champagnat } \\
\text { de Ipiales }\end{array}$ & $\begin{array}{c}\text { Colegio } \\
\text { Champagnat } \\
\text { de Pasto }\end{array}$ & $\begin{array}{c}\text { Colegio } \\
\text { Champagnat } \\
\text { de Popayán }\end{array}$ & $\begin{array}{c}\text { Colegio } \\
\text { Champagnat } \\
\text { de Cali }\end{array}$ & $\begin{array}{c}\text { Colegio } \\
\text { Champagnat } \\
\text { de Armenia }\end{array}$ & $\begin{array}{c}\text { Colegio } \\
\text { Champagnat } \\
\text { de Ibagué }\end{array}$ & $\begin{array}{c}\text { Líder } \\
\text { nacional } \\
\text { de SGC }\end{array}$ & $\begin{array}{c}\text { Porcentaje de } \\
\text { cumplimiento }\end{array}$ \\
\hline $\begin{array}{l}\text { 4. Contexto de } \\
\text { la organización }\end{array}$ & 67,3 & 47,8 & 70,0 & 90,2 & 57,0 & 71,5 & 79,2 & 69 \\
\hline 5. Liderazgo & 68,9 & 53,2 & 63,2 & 90,2 & 79,7 & 73,3 & 79,6 & 62,8 \\
\hline 6. Planificación & 71,7 & 19,9 & 56,7 & 75,7 & 40,0 & 63,3 & 54,0 & 54,4 \\
\hline 7. Apoyo & 69,5 & 65,0 & 60,0 & 85,0 & 75,0 & 70,0 & 66,0 & 80,8 \\
\hline 8. Operación & 65,5 & 54,4 & 60,0 & 91,9 & 78,8 & 73,8 & 84,6 & 72.7 \\
\hline $\begin{array}{l}\text { 9. Evaluación } \\
\text { del desempeño }\end{array}$ & 67,7 & 56,7 & 65,4 & 15,7 & 78,3 & 80,0 & 85,1 & 64.1 \\
\hline 10. Mejora & 70,0 & 56,7 & 60,0 & 16,1 & 78,3 & 76,7 & 81,1 & 62.7 \\
\hline \% cumplimiento & 68,7 & 50,5 & 62,2 & 66,4 & 69,6 & 72,7 & 75,7 & 66.5 \\
\hline
\end{tabular}

Fuente: elaboración propia.

Los resultados del análisis de la información recolectada a través de la encuesta basada en la NTC ISO 9001:2015 permiten identificar que a nivel general en los colegios privados donde se ha realizado la implementación del SGC se da un porcentaje significativo de cumplimiento respecto al contexto de la institución (numeral 4). A su vez, se observa que en los colegios San José de Armenia $(57 \%)$ e Instituto Champagnat de Pasto $(47,8 \%)$ este requisito debe ser revisado y ajustado para mejorar en su cumplimiento. Frente al requisito de liderazgo y compromiso de la alta dirección con el sistema de gestión de calidad (numeral 5), se observa que en general en la mayoría de colegios se da un cumplimiento eficiente por encima de la media, siendo el Colegio Champagnat de Pasto con $(53,2 \%)$ el de más bajo porcentaje. Respecto a la planificación (numeral 6), este es uno de los aspectos que presenta menor porcentaje de cumplimiento en la mayoría de colegios, más específicamente en los colegios San José de Armenia (40,0\%), Instituto Champagnat de Pasto $(19,9 \%$ y Colegio Champagnat de Popayán $(56 \%)$. Cabe aclarar que en gran parte esto se genera por la incorporación del enfoque basado en riesgos, que busca incluir métodos o procedimientos para la evaluación, eliminación y minimización de los riesgos. Sobre el requisito referente al apoyo (numeral 7), se observa que en la generalidad de los colegios maristas se da un resultado positivo de cumplimiento, contándose con infraestructura adecuada, personas y recursos para una buena operación y funcionamiento del SGC. Respecto a la operación (numeral 8), se evidencia que los colegios maristas presentan un buen porcentaje de cumplimiento de los requerimientos de este numeral, como por ejemplo el Colegio San Luis Gonzaga de Cali, que presenta un nivel de cumplimiento de $91.9 \%$. En 
cuando a la evaluación del desempeño (numeral 9), se observa que salvo el Colegio San Luis Gonzaga de Cali, que presenta un porcentaje de cumplimiento de $15.7 \%$, el restante de Colegios dan un buen cumplimiento a este requisito, como es el caso del Colegio Champagnat de Ibagué, con un nivel de cumplimiento de $80.0 \%$, sin embargo, es necesario progresar en el seguimiento al desempeño del personal y en el análisis de datos de las herramientas que miden la satisfacción del cliente. En cuanto a la mejora continua (numeral 10), se observa que en la mayoría de colegios certificados se da un buen porcentaje de cumplimiento, solo en el colegio San Luis Gonzaga de Cali se presenta un balance negativo con un porcentaje de cumplimiento de $16.1 \%$, la institución debe implementar de forma continua la conveniencia, adecuación y eficacia del SGC, así como determinar las oportunidades de mejora que se presentan en el desarrollo de las distintas actividades educativas que permitan el logro eficaz de los objetivos institucionales. La tabla 2 muestra los resultados de la aplicación de la encuesta diagnóstica basada en la NTC ISO 14001:2015 en los colegios maristas de Colombia.

Tabla 2. Diagnóstico comparativo del estado de cumplimiento de los requisitos del sistema de gestión ambiental basado en la NTC ISO 14001:2015 en los colegios maristas de Colombia

\begin{tabular}{|l|c|c|c|c|c|c|c|}
\hline \multicolumn{1}{|c|}{$\begin{array}{c}\text { Requisitos de la } \\
\text { Norma }\end{array}$} & $\begin{array}{c}\text { Colegio } \\
\text { Champagnat } \\
\text { de Ipiales }\end{array}$ & $\begin{array}{c}\text { Colegio } \\
\text { Champagnat } \\
\text { de Pasto }\end{array}$ & $\begin{array}{c}\text { Colegio } \\
\text { Champagnat } \\
\text { de Popayán }\end{array}$ & $\begin{array}{c}\text { Colegio } \\
\text { Champagnat } \\
\text { de Cali }\end{array}$ & $\begin{array}{c}\text { Colegio } \\
\text { Champagnat } \\
\text { de Armenia }\end{array}$ & $\begin{array}{c}\text { Colegio } \\
\text { Champagnat } \\
\text { de Ibagué }\end{array}$ & $\begin{array}{c}\text { Cumplimento } \\
\text { del SGA en las } \\
\text { organizaciones }\end{array}$ \\
\hline $\begin{array}{l}\text { 4. Contexto de la } \\
\text { organización }\end{array}$ & 0,8 & 10,0 & 1,7 & 0,0 & 0,0 & 10,0 & 3.75 \\
\hline 5. Liderazgo & 7,8 & 4,4 & 8,9 & 0,0 & 0,0 & 10,0 & 5.18 \\
\hline 6. Planificación & 5,0 & 1,8 & 3,0 & 0,0 & 0,0 & 2,0 & 1.9 \\
\hline 7. Apoyo & 2,7 & 9,0 & 13,6 & 3,3 & 4,0 & 11,7 & 8,17 \\
\hline 8. Operación & 29,3 & 7,1 & 2,1 & 0,0 & 0,0 & 10,0 & 7.75 \\
\hline 9. Evaluación del & 41,7 & 0,0 & 13,9 & 0,0 & 0,0 & 8,0 & 10,6 \\
\hline desempeño & 15,4 & 4,6 & 7,0 & 0,5 & 0,6 & 7,8 & 5,98 \\
\hline 10. Mejora
\end{tabular}

Fuente: elaboración propia. 
De otro lado, los colegios maristas de Colombia no cuentan con un sistema de gestión ambiental basado en la NTC ISO 14001:2015. Por tal motivo, al realizar la aplicación del instrumento diagnóstico basado en esta norma en los 6 colegios maristas certificados con la NTC ISO 9001:2008 se evidencia que el porcentaje de cumplimiento es muy bajo, solo se alcanza a evidenciar en algunos de los colegios acciones en gestión ambiental alineadas al proyecto PRAE, pero las instituciones no han identificado aspectos e impactos ambientales y falta definir procesos que garanticen el cumplimiento de requerimientos ambientales.

A partir de los resultados obtenidos y de las necesidades de la organización, se formula la propuesta metodológica de integración de los sistemas fundamentados en las normas técnicas NTC ISO 9001:2015 y NTC ISO 14001:2015, en los seis (6) colegios maristas definidos como muestra, partiendo desde los elementos comunes que presentan estas dos normas y tomando como referente el ciclo PHVA (planear, hacer, verificar y actuar) en la configuración del sistema de gestión integrado para la institución.

En efecto, la propuesta busca utilizar como plataforma de integración el enfoque que presenta el ciclo PHVA, como lo explican Castillo y Martínez (2006). Esto quiere decir aplicar un solo planear, hacer, verificar y actuar para la organización, para lo cual es indispensable que la organización reconozca todas las variables y opciones de información de entrada, así como sus recursos financieros, para generar a partir de allí su planificación estratégica y operativa con la definición de programas necesarios para garantizar los resultados.

Se puede considerar entonces el ciclo PHVA como una herramienta de mejoramiento que aporta a la dinámica organizacional de la planificación estratégica y al seguimiento y medición logro de los objetivos organizacionales y que, como lo expresan Atehortúa, Bustamante \& Valencia (2008), contribuye al carácter integral de la gestión, genera sinergias en las actividades, permite identificar todos los elementos presentes en los procesos que favorecen la planificación y estructuración de un sistema de gestión integral, actuando para ajustar y hacer la planificación de los cambios que el sistema requiera (véase la figura 1 ).

La propuesta para la gestión integrada en los colegios maristas destaca los puntos de inflexión de la NTC ISO 9001 y la NTC ISO 14001, luego toma cada numeral y presenta distintas estrategias metodológicas para su aplicación a partir de actividades y procesos de la organización, buscando dar cumplimiento a cada uno de los requisitos. De esta forma no solo se hacen evidentes los elementos que son convergentes en estos dos sistemas y se muestran los aspectos que se pueden integrar para articular la gestión en los colegios, sino que también se brinda a la organización una herramienta práctica que facilita realizar la estructuración e implementación del sistema de gestión integrada paso por paso, optimizando esfuerzos y recursos (véase la figura 2). 
Figura 1. Definición de requisitos comunes del SGC y del SGA desde el ciclo PHVA como plataforma de integración

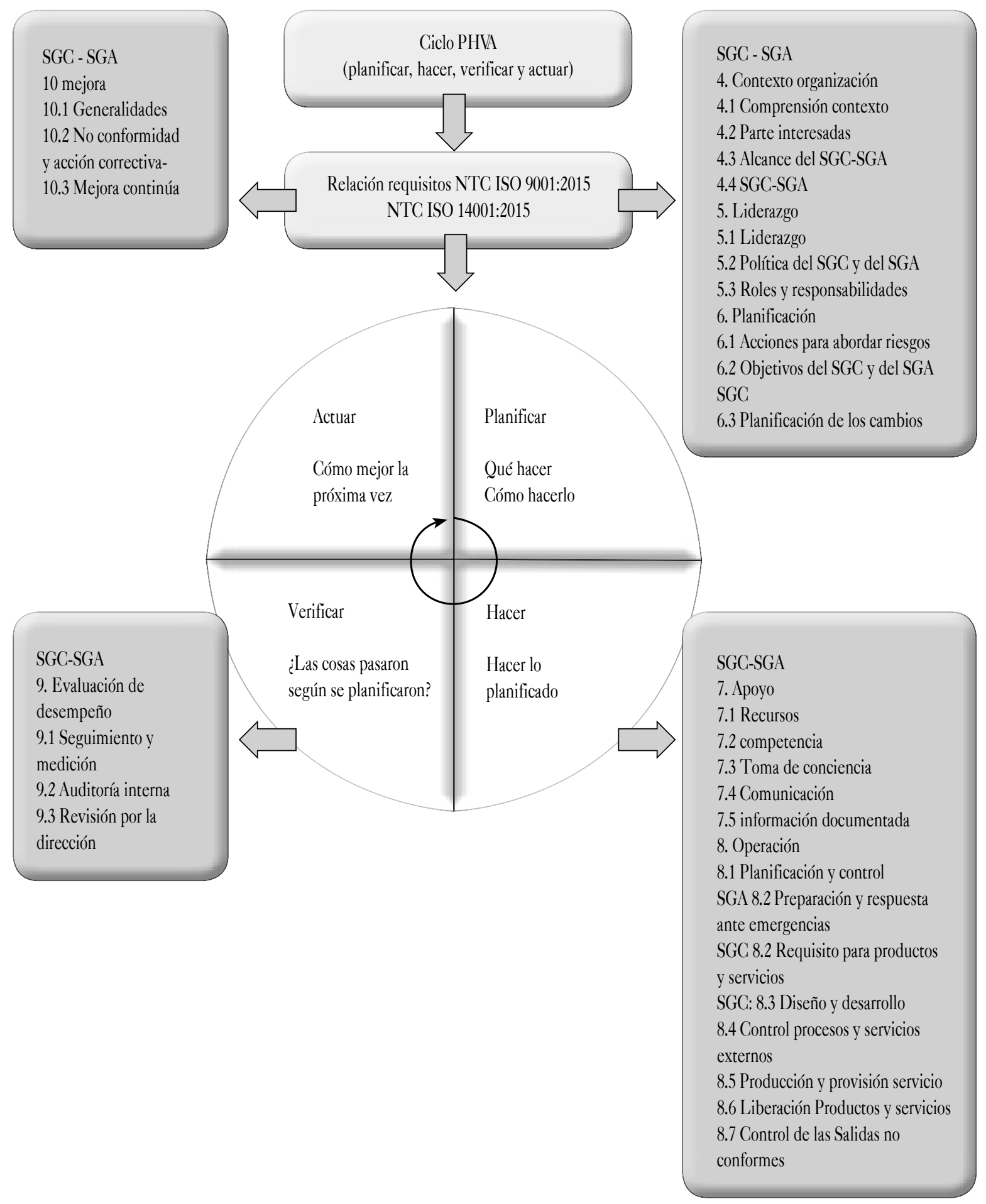

Fuente: elaboración propia. 
Figura 2. Diagrama pasos para la integración SGC-SGA

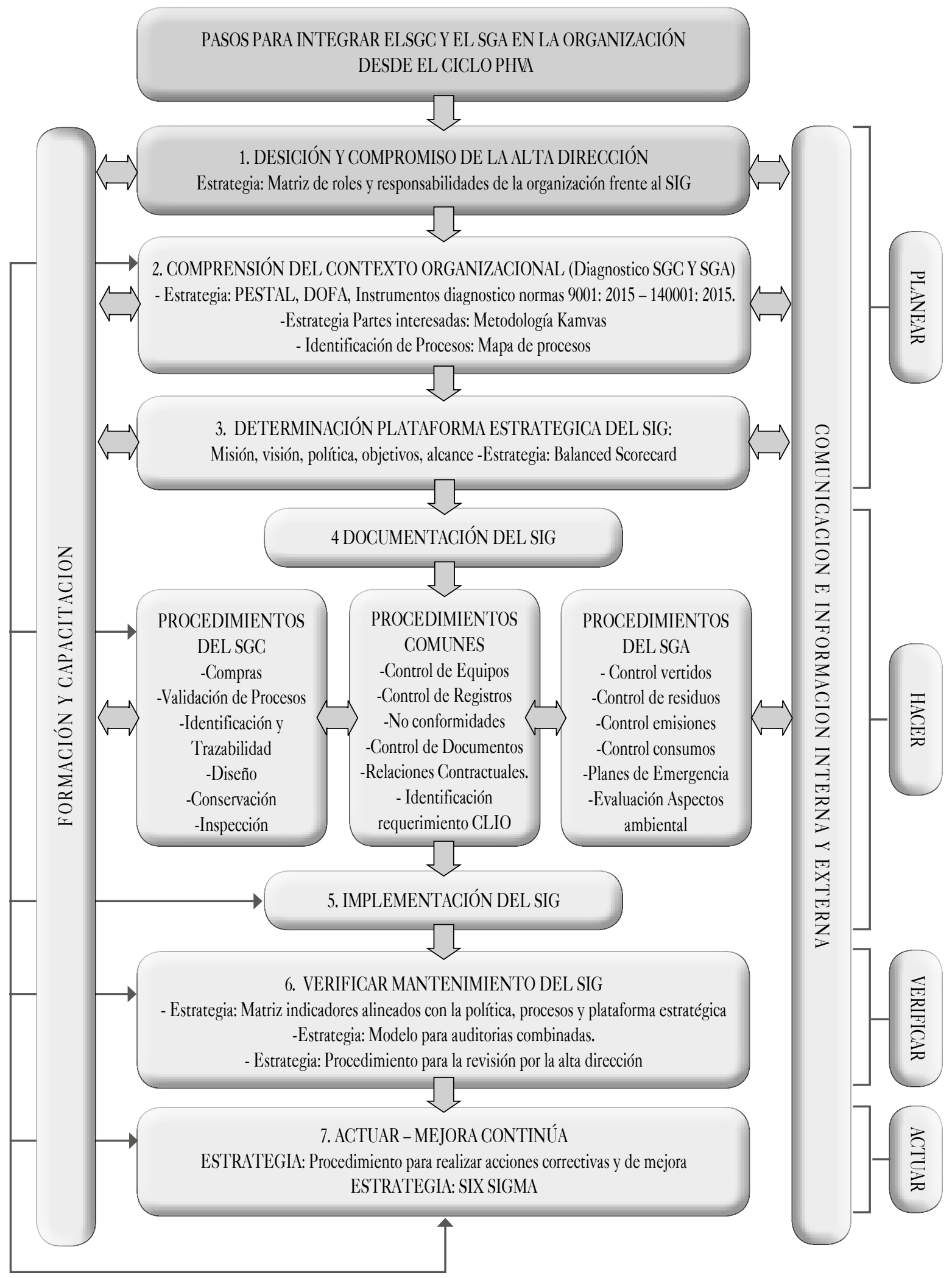

Fuente: elaboración propia. 
Con el fin de validar la propuesta se hace uso de la simulación aplicada con la participación de expertos, a través de la metodología del ábaco de Regnier, método de consulta concebido por el Dr. Francois Régnier, que tiene como propósito interrogar a expertos en un tema específico y tratar sus respuestas en tiempo real a partir de una escala basada en colores. Y que, según Godel (2010), está orientado a reducir la incertidumbre, confrontar los puntos de diferentes grupos de expertos para tomar conciencia y analizar la diversidad de opiniones que se puedan presentar en un tema concreto, realizando un registro de los resultados de esta actividad y que para efectos de la validación de la metodología permitió conocer los diferentes puntos de vista del grupo de expertos. Siguiendo el enfoque del ciclo PHVA, fue evaluada la propuesta, con el fin de validar su pertinencia y aplicabilidad en los procesos y demás actividades en la configuración el sistema integrado de gestión de la institución.

A partir del análisis de resultados obtenidos de la aplicación de la metodología ábaco de Regnier, el grupo de expertos responden a nivel general con una valoración positiva de la propuesta metodológica de integración basada en la NTC ISO 9001:2015 y la NTC ISO 14001 para los colegios maristas de Colombia, comprobándose a través de los resultados de la matriz colorimétrica, en la que predomina el color verde oscuro (muy favorable) y el color verde claro (favorable).

\section{CONCLUSIONES}

En la revisión de antecedentes se pudo evidenciar que existen investigaciones relacionadas con el tema de gestión ambiental en las organizaciones. Sin embargo, no hay investigaciones que traten directamente la integración de las normas técnicas NTC ISO 9001:2015 y la NTC ISO 14001 en el escenario educativo de colegios.

La investigación evidencia el estado actual de la organización en relación con los requerimientos de las normas técnicas ISO 9001:2015 e ISO 14001:2015, y demuestra no solo el grado de cumplimiento de los requisitos, sino también la apropiación del sistema como tal, la cultura de mejoramiento que se ha asumido en algunas de las instituciones, como es el caso del Colegio San José de Armenia, que pondera este aspecto en un 78,3\%, así como también aquellos elementos en la gestión de los sistemas que deben ser ajustados y alineados con los objetivos organizacionales, como es el caso del Colegio Champagnat de Pasto en lo referente a la planificación, requisito en el que presenta un cumplimiento del 19,9\%.

En el diagnóstico organizacional del SGA, presenta un 5,98\% de cumplimiento y permite evidenciar la necesidad de implementar un SGA en estas instituciones ya que por el tipo de servicio que se presta se genera impactos ambientales que no son atendidos suficientemente desde el PRAE, además de ser un aspecto importante que debe contemplarse dentro de la formación que se ofrece a los estudiantes en línea con la Ley 115 de febrero 8 de 1994.

Este estudio permite evidenciar los aportes que la integración de sistemas de gestión genera a organizaciones que se dedican a la prestación de servicios educativos. En este sentido, se ve cómo la adopción de sistemas de gestión basado en estas dos normas en las instituciones, si bien es cierto que implica la planificación y asignación de recursos económicos, también se logran beneficios a nivel de imagen, de cumplimiento de requisitos legales y a largo plazo de rendimiento financiero. 
La aplicación de la propuesta metodológica de integración de la NTC ISO 9001:2015 y la NTC ISO 14001:2015, desde la perspectiva del ciclo PHVA en los colegios maristas favorece que la institución pueda alcanzar estándares de calidad que aseguren su crecimiento y sostenibilidad. Por tanto, contribuye al carácter integral de la gestión, generando sinergia en las actividades e identificando elementos presentes en los procesos que favorecen la planificación y estructuración de un sistema integrado de gestión.

\section{REFERENCIAS}

Atehortúa, F., Bustamante, R. \& Valencia, J. (2008). Sistema de gestión Integral. Una sola gestión, un solo equipo. Bogotá, Colombia: Incontec.

Barrón, V. \& D'aquino, M. (2007). Proyecto y metodología de la investigación. Buenos Aires, Argentina: Maipue.

Bonilla, E. \& Rodríguez, P. (1995). La investigación en ciencias sociales. Más allá del dilema de los métodos. Bogotá, Colombia: Norma.

Castillo, D. \& Martínez, J. (2006). Enfoque para combinar e integrar la gestión de sistemas. Icontec. Bogotá, Colombia: Lemoine Editores.

Congreso de la República. Ley 115 de febrero 8 de 1994. Recuperado de http://www.mineducacion.gov. co/1621/articles-85906_archivo_pdf.pdf

Fabela, M. \& García, A. (2014). Gestión de la calidad educativa en educación superior del sector privado. Magis, Revista Internacional de Investigación en Educación, 6(13), 65-82. DOI: https://doi.org/10.11144/ Javeriana.M6-13.GCEE.
Gonzales, M. A. (2014). Integración de los sistemas de gestión de calidad, el medio ambiente y la seguridad y salud del trabajo. Revista Ciencias Holguín, 20(2). Recuperado de http://www.ciencias.holguin. cu/index.php/cienciasholguin/article/view/829/882

Godet, M. (2010). La caja de herramientas de la prospectiva estratégica. Recuperado de http//www.centrolindavista.org.mx/archivos_index/caja_de_herramientas. pdf

Hernández, S. (2014). Fases y momentos del proceso de investigación. Recuperado de https://prezi.com/ ac9n0dyy2od0/fases-y-momentos-del-proceso-deinvestigacion/

Heras, S., Merce, B. \& Casadesus F. (2007). La integración de sistemas basados en estándares internacionales: resultado de un estudio empírico realizado en la CAPV. Revista de Dirección y Administración de Empresas, 14, 155-174.

Institución educativa Champagnat Pinares de Oriente. (2017). Proyecto educativo institucional. Meta. Recuperado de http://arquidiocesisdevillavicencio.org. co/2017/01/05/institucion-educativa-champagnatpinares-de-oriente/

Kaplan \& Norton Gestiopolis. (2009).¿¿quées el balanced scorecard y para qué sirve? Recuperado de https:// www.gestiopolis.com/que-es-el-balanced-scorecard-y-para-que-sirve/

MEN (2010). Política educativa "Educación de calidad para la prosperidad". Recuperado de http://www. mineducación.gov.co/cvn/1665/articles-237397_archivo_pdf.pdf. 
Martín, J. (2017). Estudia tu entorno con un PEST-E. Obtenido de Internacional Business. Recuperado de https://www.cerembs.co/blog/estudia-tu-entorno-con-un-pest-el.

Ministerio de Educación. (2007). La gestión educativa es la vía al mejoramiento de la educación. Recuperado de https:/www.mineducacion.gov.co/1621/article-137440.html

NTC ISO 9000: 2015. (2015). Sistema de gestión de la calidad y fundamentos y vocabulario. Icontec internacional. Bogotá, D. C. Recuperado de https://www. ramajudicial.gov.co/documents/5454330/14491339/ d2.+NTC+ISO+9000-2015.pdf/ccb4b35c-ee6344b5-ba1e-7459f8714031

NTC ISO 9001:2015 (2015). Sistemas de gestión de la calidad. Requisitos. Icontec. Bogotá, D. C. Recuperado de http://www.minvivienda.gov.co/Documents/Sobre\%20el\%20Ministerio/Sistemas-de-Gestion/ NTG_ISO_9001_2015.pdf

NTC ISO 14001:2015. Sistema de Gestión Ambiental. Requisitos con orientación para su uso. Icontec. Bogotá. Recuperado de https://escuelajudicial.ramajudicial. gov.co/sites/default/files/NORMA_14001.pdf

Quiroga, C. \& Rodríguez, L. (2014). Tendencias, enfoques y desafios de los sistemas de aseguramiento de la calidad en la educación superior en el periodo comprendido entre el año 2005 y el 2013. Los casos de Colombia, Chile, Argentina, México, España, Finlandia y Estados Unidos. (Tesis de maestría). Pontificia Universidad Javeriana. Bogotá, Colombia

Sampieri, R., Fernández C. \& Baptista, P. (2010). Metodología de la Investigación. 5. ${ }^{\text {a }}$ ed. México, México D.F.: MacGraw-Hill.
Unesco. (2006). Oficina regional de la educación de la Unesco para América Latina y el Caribe. Recuperado de http://www.unesco.org/new/es/santiago/regionalbureau-for-education/

Valois, N. D. (2015). Integralidad de la gestión desde la transdisiplinariedad. Revista Signos, 7(1), 15-30. 